\title{
A limited-capacity response process in absolute identification
}

\author{
SHUJI MORI \\ University of British Columbia, Vancouver, British Columbia, Canada
}

\begin{abstract}
In two absolute identification experiments, the dependency of the current response, $R_{n}$, on the immediately preceding stimulus, $S_{n-1}$, and response, $R_{n-1}$, was measured by means of multivariate information transmission (see McGill, 1954). In these experiments, the amount of stimulus information available to subjects, measured as the amount of information transmission from a current stimulus, $S_{n}$, to $R_{n}$, was manipulated. The magnitude of the dependency of $R_{n}$ on $S_{n-1}$ and $R_{n-1}$ was inversely proportional to that of information transmission from $S_{n}$ to $R_{n}$, supporting the argument of Ward and Lockhead (1971) that the less stimulus information the subjects get, the more their responses will be likely to depend on previous stimuli and responses. Interestingly, the sum of information transmission from $\mathrm{S}_{n}, \mathrm{~S}_{n-1}$, and $\mathrm{R}_{n-1}$ to $\mathrm{R}_{n}$ was always about 2.5 bits, without respect to the variance of each term. This result could have arisen from the operation of a limited-capacity response process.
\end{abstract}

Among the many sources of response variability in absolute identification, sequential dependencies on previous stimuli and responses have been studied quite often (see Helson, 1964; Mori, 1988; Ward \& Lockhead, 1970, 1971). In earlier work, Garner (1953) and McGill (1957) measured sequential dependencies by calculating the amount of information transmission from an immediately preceding stimulus $\left(S_{n-1}\right)$ or response $\left(R_{n-1}\right)$ to $R_{n}$; this was done with the use of multivariate information transmission (McGill, 1954). In general, the information measures are nonmetric measures of given probability distributions, so that they can capture the probabilistic and/or categorical nature of stimulus and response sequences, which has been reported to affect the sequential dependencies in category judgment and absolute identification (Mori, 1988; Parducci, 1965; Ward \& Lockhead, 1971). Although it has been reported that the sequential dependencies also have metric properties (Luce \& Green, 1974), Garner and McGill (1956) showed that information analyses were in many respects similar to metric analyses such as the analysis of variance and had greater generality as a statistical tool.

The advantages of using multivariate information transmission as the measure of sequential dependencies are

Experiment 2 was conducted by the author as part of a master's thesis at Kyoto University. The data were previously reported in a somewhat different form at the annual meeting of the Japanese Psychological Association, Tokyo, October 1986, and the Japanese Basic Psychological Association, Kobe, May 1987. I wish to express my sincere gratitude to Lawrence $M$. Ward for his valuable comments on the manuscript. I thank Odie Geiger for her helpful reading of the manuscript. I would also like to thank Shinya Nishida for building the apparatus described in Experiment 1, and Masahiro Ishida and Keisuke Ido for taking part in these experiments. This research was partly supported by a grant from the Natural Sciences and Engineering Research Council of Canada to Lawrence $\mathbf{M}$. Ward. Correspondence should be addressed to the author at the Department of Psychology, University of British Columbia, 2136 West Mall, University Campus, Vancouver, BC V6T 1Y7, Canada.
(1) that the amounts of stimulus information and of sequential dependencies are estimated with the same equation and (2) that the unit of measure of both variables is the same, so that direct comparisons can be made between these amounts. Using loudness of pure tones presented in white noise, McGill (1957) showed that as the signalto-noise ratio increased, a larger amount of information transmission from $S_{n}$ to $R_{n}$ and a smaller amount of information transmission from $R_{n-1}$ to $R_{n}$ was obtained. Garner (1953) reported similar results for the dependency of $R_{n}$ on $S_{n-1}$. These and other results stimulated Ward and Lockhead (1971) to propose the general principle of psychophysical judgment that the less stimulus information the subject gets, the more likely his current response will be to depend on previous stimuli and responses.

Partly because of the lack of metric character mentioned above, the use of information analysis in psychophysical studies has declined dramatically since Garner (1962), and very little additional work has been done to explore the provocative findings regarding the relationship of information transmission from $S_{n}, S_{n-1}$, and $R_{n-1}$ to $R_{n}$. In particular, $I$ know of no reports in which the amounts of information transmission from $S_{n}, S_{n-1}$, and $R_{n-1}$ to $R_{n}$ were calculated simultaneously, on the basis of the same data. The simultaneous calculation of the amounts of information transmission from all of these sources to $R_{n}$ should reveal additional properties of the psychophysical judgment process.

With this purpose, I conducted experiments in which the amount of stimulus information available to the subjects was manipulated. It could be predicted from the results of Garner (1953) and McGill (1957), as well as from the informal model of Ward and Lockhead (1971), that the amount of sequential dependency on either $S_{n-1}$ or $R_{n-1}$ would be inversely proportional to the amount of information transmission from $S_{n}$ to $R_{n}$. However, there is no basis in previous work for anticipating the nature 
of interactions between $S_{n-1}$ and $R_{n-1}$ in their effect on $\mathbf{R}_{n}$, nor for predicting limits to the total multivariate information transmission obtained. A simple extrapolation from the notion of channel capacity (see Miller, 1956) would suggest that such limits might exist, but this gives little indication of what they might be.

\section{EXPERIMENT 1}

The task in Experiment 1 was an absolute identification task involving 10 horizontal line lengths $(1=$ shortest, $10=$ longest) and feedback. The amount of stimulus information available to the subject was manipulated by varying the luminance of the lines.

\section{Method}

Subjects. Three male graduate students of Kyoto University, one of whom was the author, took part in this experiment. All subjects had normal or corrected-to-normal visual acuity.

Stimuli and Apparatus. The 10 stimuli were horizontal lines displayed on the face of a cathode-ray tube (Iwazu SS5215, P31 phosphor), which was viewed from a distance of $1.2 \mathrm{~m}$ through a modified two-channel tachistoscope (Scientific Prototype Model $\mathrm{N}-1000$ ). The lengths of the lines were $3.00,3.27,3.54,3.85,4.18$, $4.53,4.92,5.36,5.83$, and $6.32 \mathrm{~cm}$. Presentation was controlled by a digital computer (Sharp MZ2200) with a D/A converter (DAC-UP8BC) and an analogue multiplier (Motorola MC1495L), varying the amplitude of the triangular wave fed to the $x$-axis of the oscilloscope. The exposure duration of the line was $200 \mathrm{msec}$; the duration was also controlled by the computer, with input to the $z$-axis modulated through a switching transistor.

The background field of the tachistoscope consisted of a milkwhite acrylic plate, dimly illuminated by fluorescent lights from the back. The $(x, y)$ chromaticity coordinated for the background field was $.33,0, .34$ when measured at $.4 \mathrm{~cd} / \mathrm{m}^{2}$. The luminance of the stimulus line was $2 \mathrm{~cd} / \mathrm{m}^{2}$; it was changed through placement of a neutral density filter in front of the stimulus field. The transmittances of filters used in this experiment were $12.5 \%$, $18.75 \%, 25 \%, 37.5 \%, 50 \%$, and $100 \%$ (no filter). Thus, the filter altered stimulus contrast as well as stimulus luminance, since the background field was of constant (dim) luminance. During the experiment the room was dark; the luminance was less than $3 \mathrm{~cd} / \mathrm{m}^{2}$.

Procedure. At the onset of each trial, the subjects fixated a tiny red point, which was presented for $1 \mathrm{sec}$ with a warning buzzer, $1 \mathrm{~cm}$ above the center of the viewing field where the line was to appear. Immediately after the point disappeared, a horizontal line was presented. The subjects were told to identify the length of the line. On each trial, the verbal responses given by the subjects were entered into the microcomputer by the experimenter, who also indicated the correct response.

Before the main experiment, all subjects received four 1-h practice sessions. Each subject made 300 responses ( 30 trials per stimulus) per session, for 3 sessions of all 6 luminance conditions in counter-balanced orders. A 10-min dark-adaptation period and a 10-trial practice preceded each session of the main experiment. Each session was composed of five blocks ( 60 trials per block); a short rest was given between blocks if requested for by the subjects. All trials were self-paced.

Analysis of multivariate information transmission. The equations used to calculate multivariate information transmission are based on the work of McGill (1954) and Garner (1962). The information transmission used in psychology is usually assumed to have one input and one output-for example, current stimulus $\left(S_{n}\right)$ and response $\left(R_{n}\right)$, respectively. This is denoted by $U\left(R_{n}: S_{n}\right)$, which is defined as

$$
\mathbf{U}\left(\mathbf{R}_{n}: \mathbf{S}_{n}\right)=\mathbf{U}\left(\mathbf{S}_{n}\right)+\mathrm{U}\left(\mathbf{R}_{n}\right)-\mathbf{U}\left(\mathbf{S}_{n}, \mathbf{R}_{n}\right),
$$

where $U\left(S_{n}\right)$ represents input information, $U\left(R_{n}\right)$ output information, and $U\left(S_{n}, R_{n}\right)$ the joint information of $S_{n}$ and $R_{n}$.

McGill (1954) developed the concept of multivariate information transmission. His equations for three inputs and one output were applied in the present study, with $S_{n}, S_{n-1}$, and $R_{n-1}$ as inputs, and $R_{n}$ as the output, thus:

$$
\begin{aligned}
\mathbf{U}\left(\mathbf{R}_{n}\right)= & \mathrm{U}\left(\mathbf{R}_{n}: \mathbf{S}_{n}\right)+\mathrm{U}\left(\mathbf{R}_{n}: \mathbf{S}_{n-1} \mid \mathbf{S}_{n}\right)+\mathrm{U}\left(\mathbf{R}_{n}: \mathbf{R}_{n-1} \mid \mathbf{S}_{n}\right) \\
& +\mathrm{U}\left(\mathbf{R}_{n}: \mathbf{S}_{n-1}, \mathbf{R}_{n-1} \mid \mathbf{S}_{n}\right)+\mathrm{U}\left(\mathbf{R}_{n} \mid \mathbf{S}_{n}, \mathbf{S}_{n-1}, \mathbf{R}_{n-1}\right) .
\end{aligned}
$$

$\mathrm{U}\left(\mathrm{R}_{n}: \mathrm{S}_{n-1} \mid \mathrm{S}_{n}\right)$ is the information transmission from $\mathrm{S}_{n-1}$ with the effect of $S_{n}$ eliminated, which can be considered a measure of the amount of sequential dependency of $R_{n}$ on $S_{n-1}$, and $U\left(R_{n}: R_{n-1} \mid S_{n}\right)$ is the information transmission from $R_{n-1}$ with the effect of $S_{n}$ eliminated, which can be considered a measure of the amount of sequential dependency on $R_{n-1}$. $U\left(R_{n}: S_{n-1}, R_{n-1} \mid S_{n}\right)$ can be considered the interaction effect of $S_{n-1}$ and $R_{n-1}$ with the effect of $S_{n}$ eliminated, and $U\left(R_{n} \mid S_{n}, S_{n-1}, R_{n-1}\right)$ is the error term. For detailed calculations, see the Appendix.

\section{Results and Discussion}

The information measures depicted in the section above were calculated separately for each subject. Although the amount of data available is minimal for the calculations of multivariate information transmission in this situation (10 stimuli, 10 preceding stimuli, and 10 preceding responses produce a 1,000 -cell confusion matrix), it is not necessary that all cells contain entries (cf. Garner, 1953), so the available data should suffice. Since the results for each subject were highly similar in all measures, they were averaged, so that each of the values reported below is based on 2,700 responses. To assess the reliability of the results, analyses of variance were performed on the measures for each subject.

Table 1 shows the values of all terms except the last one in Equation 2, as a function of transmittance of the ND filter. This indicates the relative line luminance (and/or contrast)-that is, the line luminance/contrastincreases as the transmittance of the filter increases. While $\mathrm{U}\left(\mathrm{R}_{n}\right)$ takes almost the same value at all luminances, $\mathrm{U}\left(\mathrm{R}_{n}: \mathrm{S}_{n}\right)$ increases reliably as the line luminance increases $[F(5,10)=89.58, p<.01]$. Thus, the manipulation of the amount of stimulus information available to the subjects was successful in this experiment.

Both $U\left(R_{n}: S_{n-1} \mid S_{n}\right)$ and $U\left(R_{n}: R_{n-1} \mid S_{n}\right)$ decrease reliably as line luminance increases $[F(5,10)=56.67$ for $\mathrm{U}\left(\mathrm{R}_{n}: \mathrm{S}_{n-1} \mid \mathrm{S}_{n}\right)$, and $\boldsymbol{F}\left(5,10=45.60\right.$ for $\mathrm{U}\left(\mathrm{R}_{n}: \mathrm{R}_{n-1} \mid \mathrm{S}_{n}\right)$, both $p s<.01]$. Moreover, $\mathrm{U}\left(\mathrm{R}_{n}: \mathrm{S}_{n-1}, \mathrm{R}_{n-1} \mid \mathrm{S}_{n}\right)$ also decreases reliably as luminance increases $[F(5,10)=30.50$, $p<.01]$. Thus, we can conclude that the predicted inverse relation between the amounts of stimulus information available to subjects and of sequential dependencies on $S_{n-1}$ and on $R_{n-1}$ has been produced. This inverse relation is more apparent in Figure 1. Figure 1 shows the quantitative relation of the five terms in the right side of Equation 2 for all transmittance values of the neutral density filter.

In addition to this, although each term alone varies with line luminance, the sum of the first four terms equals about 2.5 bits for all values of line luminance. This is supported 
Table 1

$\mathbf{U}\left(\mathbf{R}_{n}\right), \mathbf{U}\left(\mathbf{R}_{n}: S_{n}\right), \mathbf{U}\left(\mathbf{R}_{n}: S_{n-1} \mid S_{n}\right), \mathbf{U}\left(\mathbf{R}_{n}: \mathbf{R}_{n-1} \mid S_{n}\right)$, and $\mathbf{U}\left(\mathbf{R}_{n}: S_{n-1}, \mathbf{R}_{n-1} \mid S_{n}\right)$ As a Function of Transmittance of ND Filter

\begin{tabular}{cccccc}
\hline $\begin{array}{l}\text { Transmittance } \\
\text { of ND Filter }\end{array}$ & $\mathbf{U}\left(\mathbf{R}_{n}\right)$ & $\mathrm{U}\left(\mathbf{R}_{n}: \mathbf{S}_{n}\right)$ & $\mathrm{U}\left(\mathbf{R}_{n}: \mathbf{S}_{n-1} \mid \mathbf{S}_{n}\right)$ & $\mathrm{U}\left(\mathbf{R}_{n}: \mathbf{R}_{n-1} \mid \mathbf{S}_{n}\right)$ & $\begin{array}{c}\mathrm{U}\left(\mathbf{R}_{n}: \mathbf{S}_{n-1} \text {, }\right. \\
\left.\mathbf{R}_{n-1} \mid \mathbf{S}_{n}\right)\end{array}$ \\
\hline $12.5 \%$ & 3.255 & .116 & .688 & .691 & .981 \\
$18.75 \%$ & 3.280 & .462 & .646 & .598 & .726 \\
$25.0 \%$ & 3.301 & .852 & .511 & .523 & .453 \\
$37.5 \%$ & 3.308 & 1.690 & .283 & .283 & .130 \\
$50.0 \%$ & 3.310 & 1.824 & .275 & .267 & .066 \\
$100.0 \%$ & 3.316 & 2.080 & .244 & .236 & .011 \\
\hline
\end{tabular}

by analysis of variance, in that there are no significant differences of the sum of the four terms across the lineluminance conditions $[F(5,10)=1.96]$. Furthermore, 2.5 bits corresponds to the value of what has been called channel capacity (Baird \& Noma, 1978; Garner, 1962; Miller, 1956).

Channel capacity is a term borrowed from communications theory (Shannon \& Weaver, 1964); it represents an upper limit on the ability of a single information channel to transmit information. In absolute identification experiments, it has generally been found that as the amount of available stimulus information increases, the amount of information transmission increases at first but then levels off at some asymptotic value. This asymptotic value is regarded as a channel capacity for the human information channel, and it has been thought to represent a limit of the perceptual process in the transmission of stimulus information (Braida \& Durlach, 1972; Miller, 1956; Norwich, 1981).

However, it is unlikely that the value of 2.5 bits shown in Figure 1 arose only from limits of the perceptual process, because it includes the effects of $S_{n-1}$ and $R_{n-1}$ on $R_{n}$. Thus, it is possible that 2.5 bits represents an overall limit on total information transmission from $S_{n}, S_{n-1}$, and $R_{n-1}$ to $R_{n}$, rather than a simple channel capacity. Yet

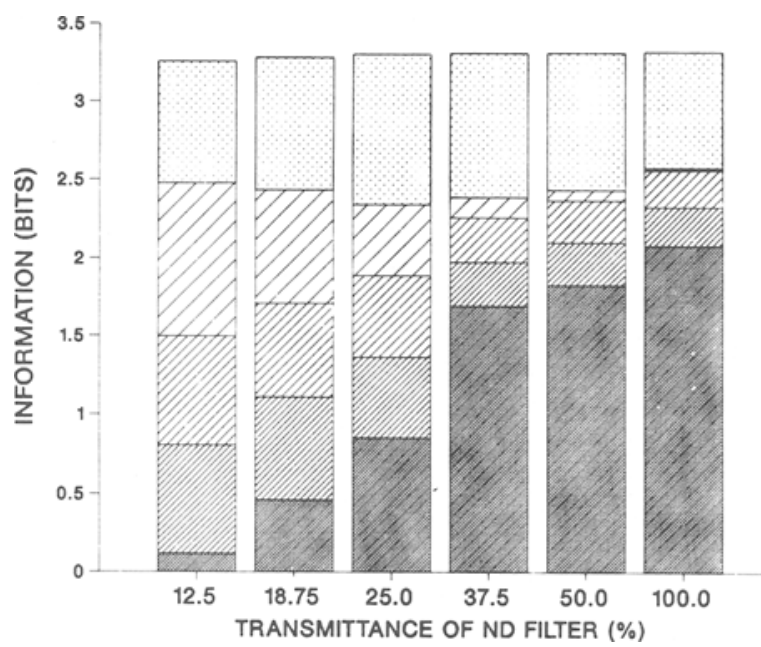

Figure 1. The quantitative relation of the five terms in the right side of Equation 2 as a function of transmittance of the ND filter. From bottom to top, $U\left(\mathbf{R}_{k}: S_{n}\right), \mathbf{U}\left(\mathbf{R}_{n}: S_{n-1} \mid S_{n}\right), \mathbf{U}\left(\mathbf{R}_{n}: \mathbf{R}_{n-1} \mid S_{n}\right)$, $\mathbf{U}\left(\mathbf{R}_{n}: S_{n-1}, \mathbf{R}_{n-1} \mid S_{n}\right)$, and $U\left(\mathbf{R}_{n} \mid S_{n}, S_{n-1}, \mathbf{R}_{n-1}\right)$. no similar observation has been reported as far as I know, so that more studies are needed for further discussion of this point. For this purpose, previously reported data (Mori, 1986, 1988) were reanalyzed, as is reported here in Experiment 2.

\section{EXPERIMENT 2}

Experiment 2 differed from Experiment 1 primarily in the use of a no-feedback condition. Eriksen (1958) first reported that giving subjects trial-by-trial feedback about their performance increased information transmission to a small but noticeable degree. Ward and Lockhead (1970, 1971) reported similar results. Braida and Durlach (1972) reported that performance was improved $20 \%$ when feedback was given in an experiment on the absolute identification of loudness. Thus, feedback typically increases the amount of information transmission in absolute identification experiments. The method of Experiment 2 is summarized here and detailed in Mori (1986, 1988).

\section{Method}

There were five conditions in this experiment: four of them came from the combination of the two stimulus information conditions (high and low) and the two feedback presentation conditions (feedback and no feedback), and the other one was a guessing condition, in which the subjects were given no stimulus information but did receive feedback after their responses in every trial (Mori, 1988).

Subjects. Twelve undergraduate and graduate students at Kyoto University ( 9 males and 3 females) took part in Experiment 2. All subjects had normal or corrected-to-normal visual acuity. Three subjects were assigned to high-information feedback condition and to the guessing condition, and 2 subjects to each of the other conditions.

Stimuli and Apparatus. The 10 stimuli were horizontal black lines centered in a $20 \times 20 \mathrm{~cm}$ white viewing field $\left(15 \mathrm{~cd} / \mathrm{cm}^{2} \mathrm{lu}-\right.$ minance) on the display of a computer, which was viewed from a distance of $87 \mathrm{~cm}$ through a tachistoscope-like box, whose inside was painted black. The display was also used to present the fixation point and trial number, and the exposure duration and time sequence of presentation were controlled by the computer. The stimulus information was manipulated by both the stimulus range and the exposure duration as follows:

1. High-information conditions: The lengths of the lines ranged from 2.0 to $4.7 \mathrm{~cm}$ in $.3-\mathrm{cm}$ steps, and the exposure duration was $1,000 \mathrm{msec}$.

2. Low-information conditions: The lengths of the lines were 2.0, $2.15,2.3,2.45,2.6,2.75,2.9,3.05,3.35$, and $3.65 \mathrm{~cm}$, and the exposure duration was $200 \mathrm{msec}$.

Procedure. The procedure of the feedback conditions was similar to that of Experiment 1, except that both the subject's response and the correct response were presented on the display. The only 
difference between the feedback and no-feedback conditions was that the correct response was not presented in the no-feedback conditions.

The guessing condition was identical to the feedback condition, except that, instead of a horizontal line, a $22 \times 22 \mathrm{~cm}$ black square was presented $500 \mathrm{msec}$ after the fixation point disappeared in every trial, and the subjects were instructed that a horizontal line would be presented immediately after the fixation point disappeared and that they should identify its length (see Mori, 1988 for details).

Each subject made 300 responses ( 30 trials per stimulus) per session for 3 sessions. There was one practice session before the main experiment.

\section{Results and Discussion}

As with Experiment 1, the information measures were calculated for each subject and averaged over the subjects in each condition, so that each point of the figures below is based on 2,700 responses in the guessing and in the high-information feedback conditions, and on 1,800 in the other conditions. For the guessing condition, in which no stimulus was presented, the feedback value was substituted for the stimulus value in the calculation. Statistical analyses were performed on the measures for each subject.

Table 2 shows the values of the first five terms in Equation 2 for each condition. Although $U\left(R_{n}\right)$ does not differ between conditions, $U\left(R_{n}: S_{n}\right)$ varies over the conditions. To test this statistically, since $U\left(R_{n}: S_{n}\right)$ is always zero in the guessing condition, an analysis of variance was performed without the guessing condition. The analysis of variance shows a significant difference between conditions $[F(3,5)=13.72, p<.01]$, and each of the values of the four conditions was larger than zero ( $t$ test, all $p s<.05)$, which confirmed that $\mathrm{U}\left(\mathrm{R}_{n}: \mathrm{S}_{n}\right)$ varies over the conditions. $U\left(R_{n}: S_{n}\right)$ was larger in the high-information conditions than in the low-information conditions, which shows that the manipulation of the stimulus information available to subjects was successful in this experiment. Yet a two-way analysis of variance shows that although there is a significant effect of stimulus information on $\mathrm{U}\left(\mathrm{R}_{n}: \mathrm{S}_{n}\right)[F(1,5)=35.03, p<.01]$, the effect of feedback presentation and the interaction effect are not significant $[F(1,5)=1.28$ for feedback presentation, and $F(1,5)=.64$ for the interaction].

The size of $U\left(R_{n}: S_{n-1} \mid S_{n}\right)$ is inversely related to $\mathrm{U}\left(\mathrm{R}_{n}: \mathrm{S}_{n}\right)$; the difference of $\mathrm{U}\left(\mathrm{R}_{n}: \mathrm{S}_{n-1} \mid \mathrm{S}_{n}\right)$ between conditions is significant $[F(4,7)=63.5, p<.01]$. Thus an inverse relation between $U\left(R_{n}: S_{n}\right)$ and $U\left(R_{n}: S_{n-1} \mid S_{n}\right)$ also holds in this experiment. A two-way analysis of variance reveals significant effects of both stimulus information $[F(1,5)=44.81, p<.01]$ and feedback presentation $[F(1,5)=34.81, p<.01]$, and no interaction effect $[F(1,5)=.01] . \mathrm{U}\left(\mathrm{R}_{n}: \mathrm{R}_{n-1} \mid \mathrm{S}_{n}\right)$ does not show such a consistent change over conditions, and there is no significant difference between conditions $[F(4,7)=1.64]$. However, the guessing condition differs from the mean of the other conditions [one-tailed $T$ test: $T(10)=2.871, p<.01$ ] So $U\left(R_{n}: R_{n-1} \mid S_{n}\right)$ may be said to show an inverse relationship with $\mathrm{U}\left(\mathbf{R}_{n}: \mathrm{S}_{n}\right)$, although the tendency is not as apparent as it is for $U\left(R_{n}: S_{n-1} \mid S_{n}\right)$. An inverse relation can also be observed between $U\left(R_{n}: S_{n}\right)$ and $\mathrm{U}\left(\mathrm{R}_{n}: \mathrm{S}_{n-1}, \mathrm{R}_{n-1} \mid \mathrm{S}_{n}\right)$; the difference of $\mathrm{U}\left(\mathrm{R}_{n}: \mathrm{S}_{n-1}, \mathrm{R}_{n-1} \mid \mathrm{S}_{n}\right)$ between conditions is significant $[F(4,7)=28.41, p<$ $.01]$. A two-way analysis of variance shows a significant effect of stimulus information $[F(1,5)=30.69$, $p<.01]$, but neither the effect of feedback $[F(1,5)=$ $1.38]$ nor the interaction effect $[F(1,5)=1.15]$ was significant.

Figure 2 shows the quantitative relation of the five terms in right side of Equation 2. As in Experiment 1, the sum of the first four terms equals about 2.5 bits in all conditions, although it does vary somewhat across conditions $[F(4,7)=7.22, p<.05]$.

As we have seen, this experiment replicated all of the major results of Experiment 1, except for the details of the inverse relation of $\mathrm{U}\left(\mathrm{R}_{n}: \mathrm{S}_{n}\right)$ and $\mathrm{U}\left(\mathrm{R}_{n}: \mathrm{R}_{n-1} \mid \mathrm{S}_{n}\right)$. This may be partly because Experiment 2 included different subjects in each condition, whereas the same subjects performed each condition in Experiment 1. The magnitude of such response correlations may differ across individuals as well as with stimulus information (see Ward, 1982).

An analysis of variance showed that in Experiment 2, feedback presentation had no overall effect on $U\left(R_{n}: S_{n}\right)$, although there was a small effect (about .3 bits) of feedback in the high-information conditions. This is consistent with the results of Eriksen (1958), who found that feedback had a greater effect on information transmission in a moderately difficult condition than in a less or in a more difficult condition. Braida and Durlach (1972) reported that the improvement of subjects' performances by feedback presentation varied, depending on the subjects and stimuli. It is possible that in the low-information conditions, in which the subjects' performances were suppressed by the manipulation of stimulus information, feed-

Table 2

\begin{tabular}{|c|c|c|c|c|c|}
\hline Condition & $\mathbf{U}\left(\mathbf{R}_{n}\right)$ & $\mathrm{U}\left(\mathrm{R}_{n}: S_{n}\right)$ & $\mathrm{U}\left(\mathbf{R}_{n}: \mathbf{S}_{n-1} \mid \mathbf{S}_{n}\right)$ & $\mathbf{U}\left(\mathbf{R}_{n}: \mathbf{R}_{n-1} \mid \mathbf{S}_{n}\right)$ & $\begin{array}{c}\mathrm{U}\left(\mathbf{R}_{n}: \mathbf{S}_{n-1},\right. \\
\left.\mathbf{R}_{n-1} \mid \mathbf{S}_{n}\right)\end{array}$ \\
\hline Guessing & 3.161 & .000 & .739 & .444 & 1.199 \\
\hline \multicolumn{6}{|l|}{ Low information } \\
\hline No feedback & 3.125 & 1.198 & .352 & .124 & .535 \\
\hline Feedback & 3.209 & 1.211 & .336 & .167 & .530 \\
\hline \multicolumn{6}{|l|}{ High information } \\
\hline No feedback & 3.268 & 1.860 & .246 & .224 & .193 \\
\hline Feedback & 3.310 & 2.152 & .214 & .206 & .017 \\
\hline
\end{tabular}




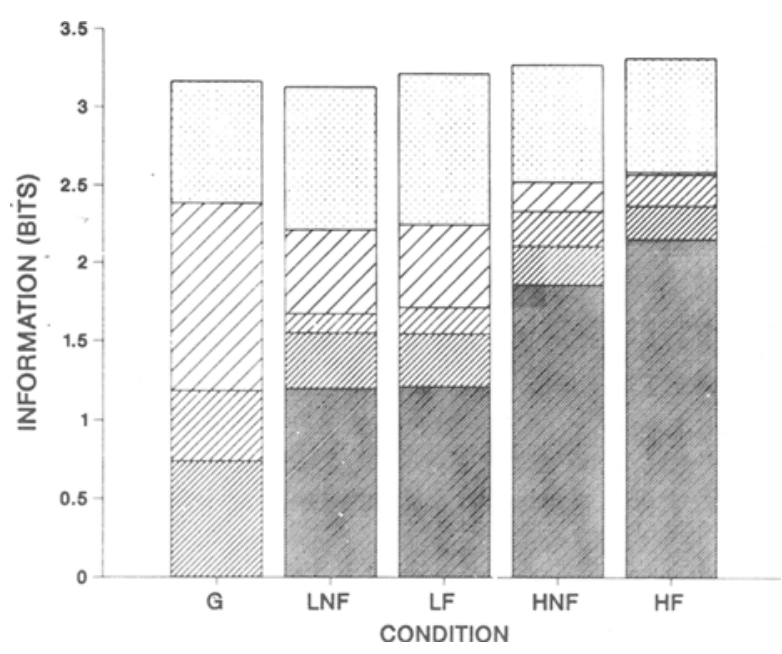

Figure 2. The quantitative relation of the five terms in the right side of Equation 2 of each condition in Experiment 2 ( $G$ = guessing, LNF = low information/no feedback, LF = low information/ feedback, HNF = high information/no feedback, HF = high information/feedback). From bottom to top, $U\left(R_{n}: S_{N}\right), U\left(R_{n}: S_{n-1} \mid S_{n}\right)$, $U\left(R_{n}: R_{n-1} \mid S_{n}\right), U\left(R_{n}: S_{n-1}, R_{n-1} \mid S_{n}\right)$, and $U\left(R_{n} \mid S_{n}, S_{n-1}, R_{n-1}\right)$.

back did not help the subjects' performances as much as it did in the high-information conditions, and the small performance difference was hidden in the individual differences in the effect of feedback.

\section{GENERAL DISCUSSION}

The original purpose in this study was to examine the inverse relation between the amount of information transmission from $S_{n}$ to $R_{n}$ and the amounts of sequential dependencies of $R_{n}$ on $S_{n-1}$ and $R_{n-1}$ by using multivariate information transmission. The results of Experiments 1 and 2 may be said to confirm this relation strongly. In both experiments, $U\left(\mathbf{R}_{n}: S_{n-1} \mid R_{n}\right)$ and $U\left(R_{n}: R_{n-1} \mid S_{n}\right)$ showed an inverse relation with $U\left(R_{n}: S_{n}\right)$, except in the case of the details for $U\left(R_{n}: R_{n-1} \mid R_{n}\right)$ in Experiment 2. In the same experiments, the results that Garner (1953) and McGill (1957) had obtained in separate experiments were replicated. In addition, an inverse relation between $\mathrm{U}\left(\mathbf{R}_{n}: \mathrm{S}_{n}\right)$ and $\mathrm{U}\left(\mathbf{R}_{n}: \mathrm{S}_{n-1}, \mathbf{R}_{n-1} \mid \mathbf{R}_{n}\right)$ was found in these experiments. These results clearly suggest that the use of multivariate information transmission gives a good picture, both of the quantitative relation between the amounts of stimulus information available to subjects, and of an information measure of the sequential dependencies of $R_{n}$ on $S_{n-1}$ and $R_{n-1}$.

Furthermore, Figures 1 and 2 show that the sum of these four terms was always approximately 2.5 bits. This finding is interesting, not only because this value was obtained without respect to the variance of the amount of each term, but also because the value of 2.5 bits reminds us of the channel capacity in an absolute identification task (Garner, 1962; Miller, 1956). However, since the value was obtained even in the guessing condition of Experiment 2, in which no line was presented, it is clear that this cannot be explained simply in terms of a limit of the perceptual process in the transmission of stimulus information.

One possible explanation arises from a consideration of response processes in absolute identification. It has been thought that psychophysical judgments are based on two processes: perceptual processes and response processes (Attneave, 1958; Curtis, 1970; Curtis, Attneave, \& Harrington, 1968; Rule \& Curtis, 1982). Roughly speaking, the perceptual processes are thought to utilize the information of stimulus intensity coded in sensory receptors (e.g., see Luce \& Green, 1978; Norwich, 1981), and the response processes are regarded as a decision process in response production (Mori, 1988; Ward, 1987). Durlach and Braida (1969) assumed that in absolute identification the subject's performance is limited by two factors due to the perceptual and response processes separately. By assuming that the two factors independently affect the variability of a category boundary, Gravetter and Lockhead (1973) successfully explained the effect of stimulus range on the absolute identification performance.

There are also some studies that reveal the effect of the response processes on channel capacity. Siegel (1972) argued that channel capacity results not only from a limit on the perceptual processes, but also from a limited capacity in the "perceptual memory" of stimuli. Note that what Siegel (1972) meant by "perceptual memory" is the subject's ability to "utilize information about items further back in the series" (Siegel, 1972, p. 124), which recalls the argument of Ward and Lockhead (1971) mentioned above. However, Ward and Lockhead's (1971) argument included the effect of previous responses, an effect that has frequently been reported to be as large as that of previous stimuli in absolute identification tasks. Since it has been reported that the response processes are a major source of sequential dependencies in absolute identification (Mori, 1988; Ward \& Lockhead, 1970, 1971), the channel capacity is thought to be affected by the response processes as well as the perceptual processes.

In addition, the results of this paper suggest that the response processes need a limited amount of information to make a response, and that this amount might be about 2.5 bits, according to Figures 1 and 2 . If this amount of information is obtained only from $S_{n}, R_{n}$ is determined solely by $S_{n}$. Otherwise, the response process tends to get information from other sources, such as $S_{n-1}$ and $R_{n-1}$, in order to make up for the lack of information, causing the sequential dependencies on them. But the total amount of information that the response processes utilize would not exceed 2.5 bits. In other words, not only the perceptual processes but also the response processes are capacitylimited.

Some previous studies support the notion of a limitedcapacity response process in absolute identification. Garner (1953) reported that perfect accuracy of absolute identification could be obtained with up to five stimulus and response categories corresponding to information transmission of 2.32 bits, but that as the number of 
categories increased beyond five categories, the amount of information transmission decreased, being only 1.62 bits for 20 categories in which stimulus information was 4.32 bits. That is, in an absolute identification task, the subject could not handle more than five categories without confusion. Siegel (1972) found that the amount of information transmission was increased by the repetitions of a given stimulus and was decreased as a function of the number of intervening trials between them. Simon (1974) demonstrated that the capacity of short-term memory was about five chunks. These results suggest that the perceptual memory of previous stimuli might affect absolute identification, although the effects are limited.

The similarity of the values of channel capacity and of the capacity of the response process may not be a mere coincidence. Norwich (1981) related the channel capacity to the firing rate of a sensory receptor by using an entropy function, and suggested that there is a limit on the sensory receptor's memory, which imposes a limit on its ability to provide information about the external world to the central nervous system. The limited capacity of the response process could be regarded as a property of the central nervous system. Thus, capacity of memory, whether through a process of a sensory receptor or of the central nervous system, limits a subject's performance in an absolute identification task. Just as channel capacity represents the maximum number of stimuli that the perceptual process can simultaneously categorize on a single sensory continuum, the capacity of the response process may represent the maximum number of category boundaries that the subject can handle in order to perform the identification task without confusion.

Although the notion of a limited-capacity response process is useful in accounting for the present results, there may be limitations to its generality. First, the present study included only 10 categories. It is possible that the capacity of the response process increases as the number of stimulus and response categories is increased. Second, according to the above explanation, there would be no (or minimal) sequential dependencies if the amount of information transmission should reach 2.5 bits. However, there are still a small number of response errors when the amount of information transmission reaches the channel capacity (Garner, 1962), and so there might still remain small but noticeable sequential dependencies.

\section{REFERENCES}

AttNeave, F. (1962). Perception and related areas. In S. Koch (Ed.), Psychology: A study of science (Vol. 4, pp. 619-659). New York: McGraw-Hill.

BAIRD, J. C., Noma, E. (1978). Fundamentals of scaling and psychophysics. New York: Wiley.

BRAIDA, L. D., \& DURLACH, N. I. (1972). Intensity perception: II. Reso lution in one-interval paradigms. Journal of the Acoustical Society of America, 51, 483-502.

CURTIS, D. W. (1970). Magnitude estimations and category judgments of brightness and brightness intervals: A two-stage interpretation. Journal of Experimental Psychology, 83, 201-208.

Curtis, D. W., Attneave, F., Harrington, T. L. (1968). A test of a two-stage model of magnitude estimation. Perception \& Psychophysics, 3, 25-31.

DURLACH, N. I., \& BraIDA, L. D. (1969). Intensity perception: I. Preliminary theory of intensity resolution. Journal of the Acoustical Society of America, 46, 372-383.

ERIKSEN, C. W. (1958). Effects of practice with or without correction on discriminative learning. American Journal of Psychology, 69, 350-358.

GaRNER, W. R. (1953). An informational analysis of absolute judgments of loudness. Journal of Experimental Psychology, 46, 373-380.

GARNER, W. R. (1962). Uncertainty and structure as psychological concepts. New York: Wiley.

GaRNER, W. R., MCGILL, W. J. (1956). The relation between information and variance analysis. Psychometrika, 21, 219-228.

Gravetter, F., \& Lockhead, G. R. (1973). Criterial range as a frame of reference for stimulus judgment. Psychological Review, 80, 203-216.

HeLson, H. (1964). Adaptation level theory: An experimental and systematic approach to behavior. New York: Harper \& Row.

LUCE, R. D., \& GREEN, D. M. (1974). The response ratio hypothesis for magnitude estimation. Journal of Mathematical Psychology, 11, $1-14$.

LuCE, R. D., \& GreEn, D. M. (1978). Two tests of a neural attention hypothesis for auditory psychophysics. Perception \& Psychophysics, 23, 363-371.

McGill, W. J. (1954). Multivariate information transmission. Psychometrika, 19, 97-116.

McGILL, W. J. (1957). Serial effects in auditory threshold judgments. Journal of Experimental Psychology, 53, 297-303.

MiLleR, G. A. (1956). The magical number seven, plus or minus two: Some limits on our capacity for processing information. Psychological Review, 63, 81-97.

MORI, S. (1986). Quantitative analysis of sequential dependencies by means of multivariate information transmitted (Rep. No. 24). Kyoto, Japan: Kyoto University, Laboratory of Psychology.

MORI, S. (1988). Two response processes in a guessing task. Perception \& Psychophysics, 44, 50-58.

NoRwich, K. H. (1981). The magical number seven: Making a "bit" of "sense." Perception \& Psychophysics, 29, 409-422.

Parducci, A. (1965). Category judgment: A range-frequency model. Psychological Review, 72, 407-418.

RULE, S. J., \& CURTIS, D. W. (1982). Levels of sensory and judgmental processing: Strategies for the evaluation of a model. In B. Wegener (Ed.), Social attitude and psychophysical measurement (pp. 107-122). Hillsdale, NJ: Erlbaum.

Shannon, C. E., \& WeAver, W. (1964). The mathematical theory of communication. Urbana: University of Illinois Press.

SiEgEL, W. (1972). Memory effects in the method of absolute judgment. Journal of Experimental Psychology, 94, 121-131.

Simon, H. A. (1974). How big is a chunk? Science, 183, 482-488.

WARD, L. M. (1982). Mixed-modality psychophysical scaling: Sequential dependencies and other properties. Perception \& Psychophysics, 31, 53-62.

WARD, L. M. (1987). Remembrance of sounds past: Memory and psychophysical scaling. Journal of Experimental Psychology: Human Perception \& Performance, 13, 27-34.

WARD, L. M., \& LoCKHEAD, G. R. (1970). Sequential effects and memory in category judgments. Journal of Experimental Psychology, 84, 27-34.

WARD, L. M., \& LOCKHEAD, G. R. (1971). Response system processes in absolute judgment. Perception \& Psychophysics, 9, 73-78.

\section{APPENDIX}

The amount of information transmission from $S_{n}$ to $R_{n}$ is defined by

where

$$
\mathbf{U}\left(\mathbf{R}_{n}: \mathbf{S}_{n}\right)=\mathbf{U}\left(\mathbf{S}_{n}\right)+\mathrm{U}\left(\mathbf{R}_{n}\right)-\mathbf{U}\left(\mathbf{S}_{n}, \mathbf{R}_{n}\right),
$$




$$
\begin{aligned}
& \mathrm{U}\left(\mathrm{S}_{n}\right)=-\Sigma P\left(\mathrm{~S}_{i}\right) \log _{2} P\left(\mathrm{~S}_{i}\right) \\
& \mathrm{U}\left(\mathrm{R}_{n}\right)=-\Sigma P\left(\mathrm{R}_{j}\right) \log _{2} P\left(\mathrm{R}_{j}\right) \\
& \mathrm{U}\left(\mathrm{S}_{n}, \mathrm{R}_{n}\right)=-\Sigma P\left(\mathrm{~S}_{i}, \mathrm{R}_{j}\right) \log _{2} P\left(\mathrm{~S}_{i}, \mathrm{R}_{j}\right) .
\end{aligned}
$$

$P\left(S_{i}\right)$ and $P\left(R_{j}\right)$ denote the probability of $S_{i}$ and of $R_{j}$, respectively, and $P\left(S_{i}, R_{i}\right)$ the joint probability of $S_{i}$ and $R_{j} . U\left(S_{n}\right)$ and $U\left(R_{n}\right)$ measure the amount of information of $S_{n}$ and of $R_{n}$, respectively, and $U\left(R_{n}, S_{n}\right)$ the amount of joint information of $S_{n}$ and $R_{n}$.

For simplicity, let us denote $n$ as the number of observations, $n_{i}$ as that of presentations of $S_{i}$, and $n_{j}$ as that of $R_{j}$ occurrences. And let us define $T=\log _{2} n, T_{i}=(1 / n) \sum n_{i} \log _{2} n_{i}$, $T_{j}=(1 / n) \sum n_{j} \log _{2} n_{j}, T_{i j}=(1 / n) \sum n_{i j} \log _{2} n_{i j}$, so we can rewrite Equation 1 as

$$
\mathrm{U}\left(\mathrm{R}_{n}: \mathrm{S}_{n}\right)=T-T_{i}-T_{j}+T_{i j}
$$

(McGill, 1954).

Next, we extend this equation to include three inputs, $S_{n}, S_{n-1}$, $R_{n-1}$. We denote the value of $S_{n-1}$ and of $R_{n-1}$ as $S_{n-1, k}$ and $\mathbf{R}_{n-1,2}$, so we define the amount of information transmission from $S_{n-1}$ to $R_{n}$ as

$$
\mathrm{U}\left(\mathrm{R}_{n}: \mathrm{S}_{n-1}\right)=T-T_{j}-T_{k}+T_{j k} .
$$

But since this measure includes the effect of $S_{n}$, we compute $U\left(R_{n}: S_{n-1}\right)$ for each value of $S_{n}$ and then average them together in order to eliminate the effect of $S_{n}$. Then we have

$$
\mathrm{U}\left(\mathrm{R}_{n}: \mathrm{S}_{n-1} \mid \mathrm{S}_{n}\right)=T_{i}-T_{i j}-T_{i k}+T_{i j k},
$$

which shows the information transmission from only $S_{n-1}$ to $R_{n}$. Similarly, the information transmission from only $R_{n-1}$ to $R_{n}$ is measured by

$$
\mathrm{U}\left(\mathbf{R}_{n}: \mathbf{R}_{n-1} \mid \mathrm{S}_{n}\right)=T_{i}-T_{i j}-T_{i}+T_{i j l} .
$$

Then we formulate the interaction effect of $S_{n-1}$ and $R_{n-1}$ in the form of the amount of information transmission from both $S_{n-1}$ and $R_{n-1}$ to $R_{n}$ as

$$
\begin{aligned}
\mathrm{U}\left(\mathrm{R}_{n}: \mathrm{S}_{n-1}, \mathrm{R}_{n-1}\right)= & -T+T_{j}+T_{k}+T_{l} \\
& -T_{j k}-T_{j l}-T_{k l}+T_{j k l} .
\end{aligned}
$$

We eliminate the effect of $S_{n}$, and we can express the interaction effect of $S_{n-1}$ and $R_{n-1}$ thus:

$$
\begin{aligned}
\mathrm{U}\left(\mathrm{R}_{n}: \mathrm{S}_{n-1}, \mathrm{R}_{n-1} \mid \mathrm{S}_{n}\right)= & -T_{i}+T_{i j}+T_{i k}+T_{i l}-T_{i j k} \\
& -T_{i j l}-T_{i k I}+T_{i j k} .
\end{aligned}
$$

Finally, $U\left(\mathbf{R}_{n}\right)$ can be expressed as follows:

$$
\begin{aligned}
\mathbf{U}\left(\mathbf{R}_{n}\right)= & \mathrm{U}\left(\mathbf{R}_{n}: S_{n}\right)+\mathrm{U}\left(\mathbf{R}_{n}: S_{n-1} \mid S_{n}\right) \\
& +\mathrm{U}\left(\mathbf{R}_{n}: \mathbf{R}_{n-1} \mid S_{n}\right)+\mathrm{U}\left(\mathbf{R}_{n}: S_{n-1}, \mathbf{R}_{n-1} \mid \mathbf{S}_{n}\right) \\
& +\mathrm{U}\left(\mathbf{R}_{n} \mid \mathbf{S}_{n}, \mathbf{S}_{n-1}, \mathbf{R}_{n-1}\right),
\end{aligned}
$$

where $U\left(R_{n} \mid S_{n}, S_{n-1}, R_{n-1}\right)$ means the error term.

(Manuscript received October 12, 1988; revision accepted for publication January $18,1989$.

\title{
Announcement
}

\author{
19th Annual Meeting of the Society for Computers in Psychology \\ Atlanta, Georgia \\ November 16, 1989
}

The 19th Annual Meeting of the Society for Computers in Psychology will be held at the Hyatt Regency Hotel in Atlanta, Georgia on November 16, 1989. As usual, this meeting will feature papers on applications of computers to all areas of psychology-experimental, clinical, and educational.

For further information, contact Paula Goolkasian, Department of Psychology, University of North Carolina at Charlotte, Charlotte, NC 28223. 